\title{
Asymptotic behavior of solutions of a semilinear heat equation with subcritical nonlinearity
}

by

\author{
Tadashi KAWANAGO \\ Department of Mathematics, Faculty of Science, Osaka University, \\ Toyonaka 560, Japan.
}

ABSTRACT. - We consider the Cauchy problem for $u_{t}=\Delta u+u^{p}$ with $1+2 / N<p$ and $(N-2) p<N+2$. We give a complete description of the asymptotic behavior of the positive solution.

RÉsumé. - Nous considérons le problème de Cauchy pour $u_{t}=\Delta u+u^{p}$ avec $1+2 / N<p$ et $(N-2) p<N+2$. On donne une description complète de comportement asymptotique de la solution positive.

\section{INTRODUCTION AND MAIN RESULT}

We study the asymptotic behavior of nonnegative solutions of the following Cauchy problem:

$$
\text { (H) }\left\{\begin{array}{l}
u_{t}=\Delta u+u^{p} \text { in } \mathbf{R}^{N} \times \mathbf{R}^{+}, \\
u(x, 0)=u_{0} \text { in } \mathbf{R}^{N} .
\end{array}\right.
$$

We assume $p>1$ and $u_{0} \geq 0, \not \equiv 0$ in $\mathbf{R}^{\mathbf{N}}$. When $u_{0} \in L^{1} \cap L^{\infty}$, Problem (H) has a unique local classical solution (see [Kawa, Proposition 2.3]), which we denote by $u\left(x, t ; u_{0}\right)$. We set

$$
t_{\max }\left(u_{0}\right):=\sup \left\{T \in \mathbf{R}^{+} ; u\left(t ; u_{0}\right) \in L^{\infty}\left((0, T) ; L^{\infty}\right)\right\} .
$$


If $t_{\max }\left(u_{0}\right)<\infty$, then we say that $u\left(t ; u_{0}\right)$ blows up in finite time. When $p \in(1,1+2 / N]$, it is well known (see e.g. [Kavi]) that all solutions of $(\mathrm{H})$ blows up in finite time. In this paper we consider the next subcritical case:

$$
1+2 / N<p \quad \text { and } \quad(N-2) p<N+2 .
$$

In spite of the simple form of Problem $(\mathrm{H})$, we need to transform the equation in order to obtain some important informations on the asymptotic behavior of solutions. Following [Kavi], we set

$$
\begin{gathered}
v\left(y, s ; u_{0}\right):=(t+1)^{1 /(p-1)} u\left(x, t ; u_{0}\right), \\
x=(t+1)^{1 / 2} y \quad \text { and } \quad t=e^{s}-1 .
\end{gathered}
$$

Then $v\left(y, s ; u_{0}\right)$ satisfies

$$
(\mathrm{TH})\left\{\begin{array}{l}
v_{s}=\Delta v+\frac{y}{2} \cdot \nabla v+\frac{v}{p-1}+v^{p} \quad \text { in } Q, \\
v(y, 0)=u_{0} \quad \text { in } \quad \mathbf{R}^{N} .
\end{array}\right.
$$

By studying Problem (TH) Kavian [Kavi] showed

$$
\left\|u\left(t ; u_{0}\right)\right\|_{\infty}=O\left(t^{-1 /(p-1)}\right) \quad \text { as } \quad t \rightarrow \infty
$$

provided $u_{0} \in H_{\rho}^{1}$ and $t_{\max }\left(u_{0}\right)=\infty$. For the definition of $H_{\rho}^{1}$, see Notations just after this section. In this paper we will extend [Kavi] and clarify the structure of space of positive solutions of $(\mathrm{H})$. Let $u_{0} \in L_{\rho}^{2} \cap L^{\infty}$. Then our main result below shows that $u\left(t ; u_{0}\right)$ is classified into one of the next three types:

Type (I): $t_{\max }\left(u_{0}\right)<\infty$, i.e. $u\left(t ; u_{0}\right)$ blows up in finite time,

Type (II): $\quad t_{\max }\left(u_{0}\right)=\infty$ and $\left\|u\left(t ; u_{0}\right)\right\|_{\infty} \sim t^{-N / 2} \quad$ as $t \rightarrow \infty$,

Type (III): $\quad t_{\max }\left(u_{0}\right)=\infty$ and $\left\|u\left(t ; u_{0}\right)\right\|_{\infty} \sim t^{-1 /(p-1)} \quad$ as $\quad t \rightarrow \infty$ and that the solution of Type (I) and the solution of Type (II) are stable and the solution of Type (III) is instable.

It is known (see e.g. [Kawa]) that if $E\left(u_{0}\right)<0$ then $u\left(t ; u_{0}\right)$ is of Type (I), where $E\left(u_{0}\right)$ is the 'energy' of $u_{0}$ defined by

$$
E\left(u_{0}\right):=\frac{1}{2}\left\|\nabla u_{0}\right\|_{2}^{2}-\frac{1}{p+1}\left\|u_{0}\right\|_{p+1}^{p+1} .
$$

Fujita $[\mathrm{F}]$ showed that if $u_{0}$ is bounded by $\varepsilon \exp \left(-a|x|^{2}\right)$ then $u\left(t ; u_{0}\right)$ is of Type (II), where $a>0$ is a constant and $\varepsilon=\varepsilon(a)>0$ is some 
small constant. In [Kawa] we gave a necessary and sufficient condition for the solution of $(\mathrm{H})$ to be of Type (II) (see Proposition 3 in Section 2), which is one of crucial results to establish our main Theorem. Haraux and Weissler [HW] observed that $(\mathrm{H})$ has a self-similar solution $w(x, t)$ of Type (III) constructed by

$$
w(x, t)=t^{-1 /(p-1)} f\left(\frac{x}{\sqrt{t}}\right),
$$

where $f \in S$ and

$$
\begin{aligned}
S:=\{f & \in H_{\rho}^{1} \cap L^{\infty} ;-\Delta f-\frac{y}{2} \cdot \nabla f \\
= & \left.\frac{f}{p-1}+f^{p} \quad \text { and. } f>0 \text { in } \quad \mathbf{R}^{\mathbf{N}}\right\} .
\end{aligned}
$$

Such a solution $w(x, t)$ is invariant by the similarity transformation:

$$
w_{\lambda}(x, t)=\lambda^{2 /(p-1)} w\left(\lambda x, \lambda^{2} t\right),
$$

namely, we have $w_{\lambda}(x, t)=w(x, t)$ for $\lambda>0$.

Now we will state our main result. Let $X:=\left\{f \in L_{\rho}^{2} \cap L^{\infty} ; f \geq\right.$ 0 in $\left.\mathbf{R}^{\mathbf{N}}\right\}$ be a closed cone of the Banach space $L_{\rho}^{2} \cap L^{\infty}$ with the norm $\|\cdot\|:=|\cdot|_{2}+\|\cdot\|_{\infty}$. We set

$$
\begin{gathered}
K:=\left\{u_{0} \in X ; t_{\max }\left(u_{0}\right)=\infty\right\} \\
B:=X-K=\left\{u_{0} \in X ; t_{\max }\left(u_{0}\right)<\infty\right\} .
\end{gathered}
$$

We denote by $\operatorname{Int}(K)$ the interior of $K$ in $X$ and by $\partial K$ the boundary of $K$ in $X$.

THEOREM 1. - We assume (1.1) Then we obtain the following:

(i) The set $K$ is an unbounded, closed convex set in $X$ and $0 \in \operatorname{Int}(K)$.

(ii) For any $u_{0} \in X-\{0\}$ there exists a unique $\tau_{0} \in \mathbf{R}^{+}$such that

$$
\left\{\begin{array}{l}
\tau_{0} u_{0} \in \partial K \\
\tau u_{0} \in \operatorname{Int}(K) \quad \text { if } \quad \tau \in\left(0, \tau_{0}\right), \\
\tau u_{0} \in B \text { if } \tau \in\left(\tau_{0}, \infty\right) .
\end{array}\right.
$$

Moreover, $G:=\left\{u_{0} \in X ;\left\|u_{0}\right\|=1\right\}$ and $\partial K$ are homeomorphic by $\left.P\right|_{G}$, where $P: X-\{0\} \rightarrow \partial K$ is the well-defined projection: $P u_{0}=\tau_{0} u_{0} \in \partial K$ in view of (1.9). 
(iii) If $u_{0} \in \operatorname{Int}(K)-\{0\}$, then we have

$$
\left\|u\left(t ; u_{0}\right)\right\|_{q} \sim t^{-(1-1 / q) N / 2} \quad \text { for } \quad q \in[1, \infty] .
$$

More precisely, for $q \in[1, \infty]$

$$
\begin{gathered}
t^{(1-1 / q) N / 2}\left\|u\left(t ; u_{0}\right)-m_{\infty}(4 \pi t)^{-N / 2} \exp \left(-\frac{|x|^{2}}{4 t}\right)\right\|_{q} \rightarrow 0 \\
\text { as } t \rightarrow \infty,
\end{gathered}
$$

where $m_{\infty}=\sup \|u(t)\|_{1} \in \mathbf{R}^{+}$.

(iv) If $u_{0} \in \stackrel{t \geq 0}{\partial} K$ then we have

$$
\left\|u\left(t ; u_{0}\right)\right\|_{q} \sim t^{N / 2 q-1 /(p-1)} \quad \text { for } \quad q \in[1, \infty] .
$$

More precisely, we obtain $\omega\left(v\left(s ; u_{0}\right)\right) \subset S$, where $\omega(v)$ is $\omega$-limit set of $v$ in $L_{\rho}^{2} \cap L^{\infty}$, i.e.

$$
\omega\left(v\left(s ; u_{0}\right)\right)=\bigcap_{t \geq 0}{\overline{\left\{v\left(s ; u_{0}\right) ; s \geq t\right\}}}^{L_{\rho}^{2} \cap L^{\infty}} .
$$

(v) If $u_{0} \in B$ then we have

$$
E\left(u\left(t ; u_{0}\right)\right) \rightarrow-\infty \quad \text { as } \quad t \rightarrow t_{\max }\left(u_{0}\right)-0 .
$$

For the Dirichlet problem in bounded domains corresponding to $(\mathrm{H})$ some similar results were established in [Li], [NST], [CL] and [G]. In this case, the solution blows up in finite time or the solution exists time-globally and converges whether to 0 or to nontrivial equilibria in $L^{\infty}$ (thus in $L^{q}$ for any $q \in[1, \infty])$ as $t \rightarrow \infty$. We remark that some methods used in their works play improtant roles in this paper by appropriate modifications. Recently, Lee and $\mathrm{Ni}$ [LN] and Wang [W] obtained some interesting necessary conditions and sufficient conditions for the solution of $(\mathrm{H})$ to exist time-globally. In particular, they treat solutions with initial values $u_{0}(x)$ decaying slowly like $|x|^{-2 /(p-1)}$ as $|x| \rightarrow \infty$.

In Section 2 we give some preliminary results in order to establish Theorem 1. In Section 3 we prove Theorem 1 and give some remarks.

Notations. $-1 . \mathbf{R}^{+}:=(0, \infty), Q:=\mathbf{R}^{\mathbf{N}} \times \mathbf{R}^{+}, Q(a, b):=\mathbf{R}^{\mathbf{N}} \times(a, b)$ and $Q[a, b):=\mathbf{R}^{\mathbf{N}} \times[a, b)$.

2. $L^{p}:=L^{p}\left(\mathbf{R}^{\mathbf{N}}\right)$ with the usual norm $\|\cdot\|_{p}:=\left(\int_{\mathbf{R}^{\mathbf{N}}}|\cdot|^{p}\right)^{1 / p}$. We denote $\|\cdot\|_{\infty, Q(a, b)}:=\|\cdot\|_{L^{\infty}(Q(a, b))}$. 
3. $\rho(x):=\exp \left(|x|^{2} / 4\right)$.

4. $L_{\rho}^{p}:=\left\{f \in L^{p} ; \int_{\mathbf{R}^{\mathbf{N}}}|f|^{p} \rho<\infty\right\}$ is a weighted $L^{p}$-space with the $\operatorname{norm}|\cdot|_{p}:=\left(\int_{\mathbf{R}^{\mathbf{N}}}|\cdot|^{p} \rho\right)^{1 / p}$.

5. $\|\cdot\|$ denotes the norm of $L_{\rho}^{2} \cap L^{\infty}$, i.e. $\|\cdot\|:=|\cdot|_{2}+\|\cdot\|_{\infty}$.

6. $H_{\rho}^{1}:=\left\{f \in H^{1}\left(\mathbf{R}^{\mathbf{N}}\right) ; \nabla f \in L_{\rho}^{2}\right\}$ is a Hilbert space with the inner product $(f, g)_{\rho}:=\int_{\mathbf{R}^{\mathbf{N}}}(\nabla f, \nabla g) \rho$ for $f, g \in H_{\rho}^{1}$.

7. $f(t)=O(g(t))$ means that $\limsup _{t \rightarrow \infty}|f(t) / g(t)|<\infty$ and $f(t) \sim$ $g(t)$ that $0<\liminf _{t \rightarrow \infty}|f(t) / g(t)| \leq \lim \sup _{t \rightarrow \infty}|f(t) / g(t)|<\infty$.

\section{PRELIMINARIES}

In this section we give some preliminary results to prove Theorem 1 .

We defined by $(1.5)$ the energy $E(u)$ for Problem $(\mathrm{H})$. We also define the energy $\hat{E}(v)$ for Problem (TH) by

$$
\hat{E}(v):=\frac{1}{2}|\nabla v|_{2}^{2}-\frac{1}{2(p-1)}|v|_{2}^{2}-\frac{1}{p+1}|v|_{p+1}^{p+1} .
$$

Proposition 1. - (i) Let $u_{0} \in X \cap H^{1}$. If $E\left(u_{0}\right)<0$ then $u_{0} \in B$.

(ii) Let $u_{0} \in X \cap H_{\rho}^{1}$. If $\hat{E}\left(u_{0}\right)<0$ then $u_{0} \in B$.

Proof. - (i) This is well-known. See e.g. the proof of [Kawa, Proposition 3.1].

(ii) See the proof of [Kavi, Theorem (1.10)].

Proposition 2. - We assume (1.1). Then the following hold.

(i) Let $b \in \mathbf{R}^{+}$. Then there exists some constant $m \in \mathbf{R}^{+}$such that for any $u_{0} \in K$ with $\left\|u_{0}\right\|_{2}+\left\|u_{0}\right\|_{\infty} \leq b$ we have $\left\|u\left(t ; u_{0}\right)\right\|_{\infty, Q} \leq m$.

(ii) The set $K$ is closed in $X$.

(iii) Let $u_{0} \in B$. Then we have (1.14).

Proof. - Using Lemma 1 below, we can prove Proposition 2 essentially by the same argument as in [G]. Therefore, we leave it to the reader.

LEMMA 1. - We assume (1.1). Let $t_{0} \in \mathbf{R}^{+}$and $u$ be a classical solution of $(H)$ on $[0, T), T>t_{0}$. Assume that

$$
\begin{gathered}
\int_{0}^{T}\left\|u_{t}\right\|_{2}^{2} d t \leq l<\infty, \\
\|u\|_{\infty, Q\left(t_{0}, T\right)}=\|u\|_{\infty, Q(0, T)} .
\end{gathered}
$$


Then there is some constant $a \in \mathbf{R}^{+}$independant of $u, u_{0}$ and $T$ (dependant of $l$ and $t_{0}$ ) such that

$$
\|u\|_{\infty, Q(0, T)} \leq a
$$

Proof. - Although the proof is similar to that of [G, Lemma], we will describe it for the sake of completeness. We proceed by a contradiction. Suppose that Lemma 1 does not hold. Then there is a sequence of solutions $u_{n}(x, t)$ of $(\mathrm{H})$ on $\left[0, T_{n}\right), T_{n}>t_{0}$ such that

$$
\begin{gathered}
\int_{0}^{T_{n}}\left\|u_{n t}\right\|_{2}^{2} d t \leq l, \\
\left\|u_{n}\right\|_{\infty, Q\left(t_{0}, T_{n}\right)}=\left\|u_{n}\right\|_{\infty, Q\left(0, T_{n}\right)},
\end{gathered}
$$

and

$$
\left\|u_{n}\right\|_{\infty, Q\left(0, T_{n}\right)} \rightarrow \infty \quad \text { as } \quad n \rightarrow \infty .
$$

Let $\left(x_{n}, t_{n}\right) \in Q\left(t_{0}, T_{n}\right)$ be a sequence such that

$$
\left|u_{n}\left(x_{n}, t_{n}\right)\right| \geq \frac{1}{2}\left\|u_{n}\right\|_{\infty, Q\left(0, T_{n}\right)} .
$$

We choose a sequence $\lambda_{n}>0$ such that

$$
\lambda_{n}^{2 /(p-1)}\left|u_{n}\left(x_{n}, t_{n}\right)\right|=1 .
$$

We remark that $\lambda_{n}$ satisfies that $\lambda_{n} \rightarrow 0$ as $n \rightarrow \infty$. We define the function $v_{n}$ by

$$
v_{n}(x, t)=\lambda_{n}^{2 /(p-1)} u_{n}\left(x_{n}+\lambda_{n} x, t_{n}+\lambda_{n}^{2} t\right) .
$$

We can easily verify that $v_{n}$ is a solution of $(\mathrm{H})$ in $Q_{n}:=Q\left(-t_{n} / \lambda_{n}^{2},\left(T_{n}-\right.\right.$ $\left.\left.t_{n}\right) / \lambda_{n}^{2}\right)$. In view of (2.7) and (2.8) we have

$$
v_{n}(0,0)=1 \text {, }
$$

$$
\left\|v_{n}\right\|_{\infty, Q_{n}}=\lambda_{n}^{2 /(p-1)}\left\|u_{n}\right\|_{\infty, Q\left(0, T_{n}\right)} \leq 2 \lambda_{n}^{2 /(p-1)}\left|u_{n}\left(x_{n}, t_{n}\right)\right|=2 .
$$

Since $\left\{v_{n}\right\}$ are uniformly bounded, $\left\{v_{n}\right\}$ are equi-continuous on every compact subset of $Q(-\infty, 0]$ (see [D] or [S]). Thus, there is a subsequence (still denoted $v_{n}$ ) and a function $v(x, t)$ such that

$$
v_{n} \rightarrow v \quad \text { in } \quad L^{\infty}(D) \quad \text { as } \quad n \rightarrow \infty
$$


where $D$ is any compact subset of $Q(-\infty, 0]$. The function $v$ is a solution of $(\mathrm{H})$ in the sense of distribution and is bounded in $Q(-\infty, 0]$. Therefore, $v$ is a classical solution of $(\mathrm{H})$. It follows from $(\mathrm{H})$ that

$$
\begin{aligned}
\int_{-t_{0} / \lambda_{n}^{2}}^{0}\left\|v_{n t}\right\|_{2}^{2} d t & =\lambda_{n}^{4 p /(p-1)-(N+2)} \int_{t_{n}-t_{0}}^{t_{n}}\left\|u_{n t}\right\|_{2}^{2} d t \\
& \leq l \lambda_{n}^{4 p /(p-1)-(N+2)} \rightarrow 0 \text { as } n \rightarrow \infty
\end{aligned}
$$

By (2.9), (2.10) and (2.11), we obtain

$$
v(0,0)=1 \quad \text { and } \quad v_{t} \equiv 0 \quad \text { in } Q(-\infty, 0] .
$$

Thus, $v$ is a nontrivial equlibrium solution of $(\mathrm{H})$. This contradicts a Liouville theorem in [GS]. The proof is complete.

Proposition 3. - Let $p>1+2 / N$ and $p_{0}:=N(p-1) / 2$. Assume that $u_{0} \in L^{1} \cap L^{\infty}, u_{0} \geq 0$, $\not \equiv 0$ in $\mathbf{R}^{\mathbf{N}}$ and $|x| u_{0}(x) \in L^{1}$. Then the following (2.13) and (2.14) are equivalent:

$$
\begin{aligned}
& t_{\max }\left(u_{0}\right)=\infty \text { and }\left\|u\left(t ; u_{0}\right)\right\|_{\infty} \sim t^{-N / 2}, \\
& \inf \left\{\left\|u\left(t ; u_{0}\right)\right\|_{p_{0}} ; t \in\left[0, t_{\max }\left(u_{0}\right)\right)\right\}<\delta_{0}
\end{aligned}
$$

where $\delta_{0}>0$ is a constant depending only on $N$ and $p$. If (2.13) holds then $u\left(t ; u_{0}\right)$ satisfies

$$
t^{(1-1 / q) N / 2}\left\|u\left(t ; u_{0}\right)-m_{\infty}(4 \pi t)^{-N / 2} \exp \left(-\frac{|x|^{2}}{4 t}\right)\right\|_{q} \rightarrow 0 \quad \text { as } \quad t \rightarrow \infty
$$

for any $q \in[1, \infty]$, where

$$
0<m_{\infty}:=\sup _{t \geq 0}\|u(t)\|_{1}=\int_{\mathbf{R}^{\mathbf{N}}} u_{0} d x+\int_{0}^{\infty} d t \int_{\mathbf{R}^{\mathbf{N}}} u(t)^{p} d x<\infty .
$$

Proof. - The equivalence of (2.13) and (2.14) follows from [Kawa, Corollary 1.1], and (2.15) with $q=\infty$ from [Kawa, Theorem 4.1]. Using [EZ, Lemma 3], we can prove (2.15) with $q=1$ in the same way as in the proof of (2.15) with $q=\infty$. By linear interpolation we obtain (2.15) for $q \in(1, \infty)$. 
Proposition 4. - Assume $p>1+2 / N$. We set

$$
W:=\left\{u_{0} \in X ; t_{\max }\left(u_{0}\right)=\infty \quad \text { and } \quad\left\|u\left(t ; u_{0}\right)\right\|_{\infty} \sim t^{-N / 2}\right\} .
$$

Then $W$ is open in $X$.

Proof. - We fix $u_{0} \in W$. Let $p_{0}=N(p-1) / 2(>1)$. It suffices to prove (2.16) $\exists \delta=\delta(N, p)>0 ; u_{1} \in X$ and $\left\|u_{1}-u_{0}\right\|_{p_{0}}<\delta \Longrightarrow u_{1} \in W$.

In view of the comparison principle we may assume without loss of generality that $u_{1} \geq u_{0}$ in $\mathbf{R}^{\mathbf{N}}$. We set $w(x, t)=u\left(x, t ; u_{1}\right)-u\left(x, t ; u_{0}\right)$ $\left(\geq 0\right.$ in $\left.\mathbf{R}^{\mathbf{N}}\right)$. Then, $w$ satisfies

$$
\begin{aligned}
w_{t} & =\Delta w+\left[w+u\left(t ; u_{0}\right)\right]^{p}-u\left(t ; u_{0}\right)^{p} \\
& =\Delta w+p w \int_{0}^{1}\left[s w+u\left(t ; u_{0}\right)\right]^{p-1} d s \\
& \leq \Delta w+2^{p-1} p w\left[w^{p-1}+\left\|u\left(t ; u_{0}\right)\right\|_{\infty}^{p-1}\right] .
\end{aligned}
$$

We set $f(t)=2^{p-1} p\left\|u\left(t ; u_{0}\right)\right\|_{\infty}^{p-1}$ and

$$
w(x, t)=W(x, t) \exp \left[\int_{0}^{t} f(s) d s\right] .
$$

Then, $f(t) \in L^{1}(0, \infty)$. The function $W$ satisfies

$$
W_{t} \leq \Delta W+C_{1} W^{p} \text {. }
$$

Here, $C_{1}=2^{p-1} p \exp \left[(p-1) \int_{0}^{\infty} f(s) d s\right] \in \mathbf{R}^{+}$. By Proposition 3, there exist $\delta=\delta(N, p)>0$ such that if $\|W(0)\|_{p_{0}}=\left\|u_{1}-u_{0}\right\|_{p_{0}}<\delta$ then we have

$$
t_{\max }\left(u_{1}\right)=\infty \quad \text { and } \quad\|W(t)\|_{\infty}=O\left(t^{-N / 2}\right) .
$$

Therefore, we obtain $\left\|u\left(t ; u_{1}\right)\right\|_{\infty} \sim t^{-N / 2}$. Hence, (2.16) holds.

LemMa 2. - Let $f, g \in S$. If $f \leq g$ in $\mathbf{R}^{\mathbf{N}}$ then $f=g$ in $\mathbf{R}^{\mathbf{N}}$.

Proof. - Our proof is very close to that of [Li, Lemma 2.2]. By integration by parts we find

$$
\int_{\mathbf{R}^{\mathbf{N}}} g^{p} f \rho=\int(\nabla f \cdot \nabla g) \rho-\frac{1}{p-1} \int f g \rho=\int f^{p} g \rho,
$$

which leads to

$$
\int_{\mathbf{R}^{\mathrm{N}}} \rho f g\left(g^{p-1}-f^{p-1}\right)=0
$$

This yields $f \equiv g$ in $\mathbf{R}^{\mathbf{N}}$. 
Proposition 5. - We assume (1.1). Let $u_{0} \in X$ and $t_{\max }\left(u_{0}\right)=\infty$. Then $u\left(t ; u_{0}\right)$ satisfies whether

$$
\left\|u\left(t ; u_{0}\right)\right\|_{\infty} \sim t^{-1 /(p-1)}
$$

or

$$
\left\|u\left(t ; u_{0}\right)\right\|_{\infty} \sim t^{-N / 2}
$$

Moreover, if (2.18) holds then we have

$$
\omega\left(v\left(s ; u_{0}\right)\right) \subset S
$$

and if (2.19) holds then we have

$$
\omega\left(v\left(s ; u_{0}\right)\right)=\{0\} .
$$

Proof. - We can verify that

$$
\left\|u\left(t ; u_{0}\right)\right\|_{q}=(t+1)^{\left(1 / q-1 / p_{0}\right) N / 2}\left\|v\left(s ; u_{0}\right)\right\|_{q} \quad \text { for } \quad q \in[1, \infty],
$$

$$
\frac{d}{d s} \hat{E}\left(v\left(s ; u_{0}\right)\right)=-\left|v_{s}\left(s ; u_{0}\right)\right|_{2}^{2}
$$

Kavian [Kavi, Theorem (1.13)] showed

$$
\left\|u\left(t ; u_{0}\right)\right\|_{\infty}=O\left(t^{-1 /(p-1)}\right) \quad\left(\Longleftrightarrow\left\|v\left(s ; u_{0}\right)\right\|_{\infty}=O(1)\right)
$$

and

$$
\omega\left(v\left(s ; u_{0}\right)\right) \subset S \cup\{0\}
$$

He proved (2.24) by using (2.23) and the method in [CL]. Once we obtain (2.24), we can derive (2.25) from the smoothing effect: $v\left(s ; u_{0}\right) \in L^{\infty}\left([\tau, \infty) ; H_{\rho}^{1} \cap C^{1}\left(\mathbf{R}^{\mathbf{N}}\right)\right)$ for $\tau>0$ and the compactness of the embedding: $H_{\rho}^{1} \cap C^{1}\left(\mathbf{R}^{\mathbf{N}}\right) \subset L_{\rho}^{2} \cap L^{\infty}$. We remark that the method in [G] is also applicable to deduce (2.24). Indeed, using Lemma 3 below, we can prove (2.24) by the same argument in the proof of Proposition 2, (i). 
Next, we will show that if (2.18) does not hold then (2.19) holds. Let $u\left(t ; u_{0}\right)$ do not satisfy (2.18). Then we have

$$
\liminf _{t \rightarrow \infty} t^{1 /(p-1)}\left\|u\left(t ; u_{0}\right)\right\|_{\infty}=0
$$

Or equivalently,

$$
\liminf _{s \rightarrow \infty}\left\|v\left(s ; u_{0}\right)\right\|_{\infty}=0
$$

Therefore, we deduce that

$$
0 \in \omega\left(v\left(s ; u_{0}\right)\right)
$$

which leads to

$$
\liminf _{t \rightarrow \infty}\left\|u\left(t ; u_{0}\right)\right\|_{p_{0}}=\liminf _{s \rightarrow \infty}\left\|v\left(s ; u_{0}\right)\right\|_{p_{0}}=0 .
$$

By Proposition 3 we obtain (2.19). Now, we see that (2.19), (2.21) and (2.26) are equivalent. Thus, (2.18) and (2.20) are also equivalent.

LEMma 3. - We assume (1.1). Let $s_{0} \in \mathbf{R}^{+}$and $v$ be a classical solution of $(T H)$ on $[0, T), T>s_{0}$. Assume that

$$
\begin{gathered}
\int_{0}^{T}\left|v_{s}\right|_{2}^{2} d s \leq l<\infty, \\
\|v\|_{\infty, Q\left(s_{0}, T\right)}=\|v\|_{\infty, Q(0, T)} .
\end{gathered}
$$

Then there is some constant $a \in \mathbf{R}^{+}$independant of $v, u_{0}$ and $T$ (dependant of $l$ and $s_{0}$ ) such that

$$
\|v\|_{\infty, Q(0, T)} \leq a
$$

Proof. - Since the proof is essentially the same as that of Lemma 1, we leave it to the reader.

\section{PROOF OF THEOREM 1 AND REMARKS}

Proof of Theorem 1. - Let $W$ be the open set in $X$ defined in the statement of Proposition 4.

(i) We already proved the closedness of $K$ (see Proposition 2). By the same argument as in [Li], we can verify that $K$ is convex. The 
unboundedness of $K$ follows from Proposition 3. Indeed, we can easily find $u_{0}^{n} \in X$ such that $\left\|u_{0}^{n}\right\|_{p_{0}}<\delta_{0}$ and $\left\|u_{0}^{n}\right\|_{\infty}>n$ for $n \in \mathbf{N}$. By Proposition $3,\left\{u_{0}^{n}\right\}$ is an unbounded sequence in $K$. We can see $0 \in \operatorname{Int}(K)$ also in view of Proposition 3.

(ii) We fix any $u_{0} \in X-\{0\}$. We set $L=\left\{\tau \in \mathbf{R}^{+} ; \tau u_{0} \in W\right\}$ and $M=\left\{\tau \in \mathbf{R}^{+} ; \tau u_{0} \in B\right\}$. The sets $L$ and $M$ are open connected sets with $L \neq \phi$ and $M \neq \phi$. Therefore, $\mathbf{R}^{+}-(L \cup M) \neq \phi$. Set $\tau_{0}=\min \left\{\mathbf{R}^{+}-(L \cup M)\right\}$ and $\tau_{1}=\max \left\{\mathbf{R}^{+}-(L \cup M)\right\}$. By the definition we have $\tau_{1} u_{0} \in \partial K, \tau u_{0} \in W$ if $\tau<\tau_{0}$ and $\tau u_{0} \in B$ if $\tau>\tau_{1}$. We will show that $\tau_{0}=\tau_{1}$. Since $\tau_{1} \tau_{0}^{-1} v\left(s ; \tau_{0} u_{0}\right)$ is a subsolution of (TH) with the initial value $\tau_{1} u_{0}$, we obtain

$$
v\left(y, s ; \tau_{1} u_{0}\right) \geq \frac{\tau_{1}}{\tau_{0}} v\left(y, s ; \tau_{0} u_{0}\right) \quad \text { in } \quad Q .
$$

Therefore, there exist $f \in \omega\left(v\left(s ; \tau_{1} u_{0}\right)\right)$ and $g \in \omega\left(v\left(s ; \tau_{0} u_{0}\right)\right)$ such that

$$
f \geq \frac{\tau_{1}}{\tau_{0}} g \text { in } \mathbf{R}^{\mathbf{N}} \text {. }
$$

By Proposition 5,

$$
\omega\left(v\left(s ; \tau_{0} u_{0}\right)\right) \cup \omega\left(v\left(s ; \tau_{1} u_{0}\right)\right) \subset S .
$$

It follows from (3.2), (3.3) and Lemma 2 that $f=g$ and $\tau_{0}=\tau_{1}$. Thus we have proved (1.9).

Now we know that the map $\left.P\right|_{G}: G \rightarrow \partial K$ is one to one and onto. Cleary, $\left(\left.P\right|_{G}\right)^{-1}$ is continuous. Thus, it suffices to show that $\left.P\right|_{G}$ is continuous. Let $\left\{x_{n}\right\}_{n \in \mathbf{N}} \subset G$ be a sequence in $X$ such that $x_{n} \rightarrow x_{0}$ in $X$ as $n \rightarrow \infty$ for some $x_{0} \in G$. We will prove

$$
P x_{n} \rightarrow P x_{0} \quad \text { in } \quad X \quad \text { as } n \rightarrow \infty .
$$

We fix a number $\lambda>1$. We can easily check

$$
\lambda\left\|P x_{0}\right\| x_{n} \rightarrow \lambda P x_{0} \quad \text { in } \quad X \quad \text { as } n \rightarrow \infty .
$$

Since $\lambda P x_{0} \in B$ and $B$ is open, $\lambda\left\|P x_{0}\right\| x_{n} \in B$ for sufficiently large $n$. Thus we obtain

$$
\sup _{n \in \mathbf{N}}\left\|P x_{n}\right\|<\infty,
$$

By (3.6) there exist a subsequence (still denoted $\left\{P x_{n}\right\}_{n}$ ) and a number $a>0$ such that

$$
\left\|P x_{n}\right\| \rightarrow a \quad \text { as } \quad n \rightarrow \infty .
$$

Vol. 13, $\mathrm{n}^{\circ} 1-1996$. 
It follows that

$$
\begin{aligned}
& \left\|P x_{n}-a x_{0}\right\| \leq\left\|P x_{n}-\right\| P x_{n}\left\|x_{0}\right\|+\|\| P x_{n}\left\|x_{0}-a x_{0}\right\| \\
& =\left\|P x_{n}\right\|\left\|x_{n}-x_{0}\right\|+\|\| P x_{n} \|-a \mid \rightarrow 0 \text { as } n \rightarrow \infty
\end{aligned}
$$

Therefore, we deduce that $a x_{0} \in \partial K$ and $a=\left\|P x_{0}\right\|$. Since $a$ is a unique constant independant of the way to choose a subsequence, we obtain (3.4).

(iii) By the proof of (ii) we can derive that $\operatorname{Int}(K)=W$. Thus we have (1.11) by Proposition 3. The estimate (1.10) follows from (1.11).

(iv) Let $u_{0} \in \partial K$. By the proof of Proposition 5 we have

$$
\left\|v\left(s ; u_{0}\right)\right\|_{q} \sim 1
$$

for $q=p_{0}$ and $q=\infty$. Since $v\left(s ; u_{0}\right)$ is bounded in $X$ for $s \geq 0$, we have

$$
\left\|v\left(s ; u_{0}\right)\right\|_{1}=O(1) .
$$

Therefore, (3.9) actually holds for any $q \in[1, \infty]$. Combining (2.22) and (3.9), we deduce (1.12). We obtain from (3.9) and Proposition 5 that $\omega\left(v\left(s ; u_{0}\right)\right) \subset S$.

(v) We have already obtained (1.14) (see Proposition 2).

Finally we give two remarks concerning Theorem 1 .

Remark 1. - We observe that the Haraux-Weissler self-similar solution $w(t)$ given in (1.6) satisfies $\|w(t)\|_{q} \rightarrow \infty$ as $t \rightarrow \infty$ for $q \in$ $[1, N(p-1) / 2)$. This fact also leads to the unboundedness of $K$ in $X$.

Remark 2. - With respect to (iv) of Theorem 1 we have the following result:

Proposition. - Assume (1.1) and $p \in$ N. Then for any $u_{0} \in \partial K$ the set $\omega\left(v\left(s ; u_{0}\right)\right) \subset S$ consists of only one element, i.e. $\omega(v)=\{\varphi\}$, where $\varphi$ is an element of $S$.

Outline of the proof of Proposition. - We will apply the method by Simon [Si]. Let $\varphi \in \omega\left(v\left(s ; u_{0}\right)\right)$ for $u_{0} \in \partial K$. We will derive $\omega(v)=\{\varphi\}$. We set $\mathcal{E}(u):=\hat{E}(u+\varphi)$ (see (2.1) for the definition of $\hat{E}$ ) and $w(s):=v\left(s ; u_{0}\right)-\varphi$. Then $w(s)$ satisfies

$$
\begin{gathered}
w_{s}=\mathcal{M}(w), \\
\frac{d}{d s} \mathcal{E}(w(s))=-\left|w_{s}\right|_{2}^{2} .
\end{gathered}
$$


where we set

$$
\begin{aligned}
\mathcal{M}(w): & =\Delta(w+\varphi)+\frac{y}{2} \cdot \nabla(w+\varphi)+\frac{w+\varphi}{p-1}+(w+\varphi)^{p} \\
& =\Delta w+\frac{y}{2} \cdot \nabla w+\frac{w}{p-1}+(w+\varphi)^{p}-\varphi^{p}
\end{aligned}
$$

Let $H_{\rho}^{2}:=\left\{f \in H_{\rho}^{1} ; \nabla f \in H_{\rho}^{1}\right\}$. The space $H_{\rho}^{2}$ is a Hilbert space with the norm $|f|_{\rho}:=\left(\sum_{i, j=1}^{N}\left|\partial^{2} f / \partial y_{i} \partial y_{j}\right|_{2}^{2}\right)^{1 / 2}$ for $f \in H_{\rho}^{2}$. Since $p \in \mathbf{N}$ and $H_{\rho}^{2} \hookrightarrow L_{\rho}^{2 p}$ (cf. [Kavi, Lemma 2.1]), the map $\mathcal{M}: H_{\rho}^{2} \rightarrow L_{\rho}^{2}$ is analytic. We set $L:=d \mathcal{M}(0)$. We have

$$
L w=\Delta w+\frac{y}{2} \cdot \nabla w+\frac{w}{p-1}+p \varphi^{p-1} w .
$$

We define $A: L_{\rho}^{2} \rightarrow L_{\rho}^{2}$ by

$$
A w:=\Delta w+\frac{y}{2} \cdot \nabla w
$$

with $D(A)=H_{\rho}^{2}$. We know (see [Kavi, Lemma 2.1]) that $-A$ is a positive self-adjoint operator with compact inverse. Since $\varphi \in L^{\infty}$, there exists a complete ortho-normal system $\left\{\psi_{j}\right\}_{j=1}^{\infty}$ for $L_{\rho}^{2}$ which consists of eigenfunctions of the operator $L$. We denote by $\Pi$ the orthogonal projection of $L_{\rho}^{2}$ onto the (finite-dimensional) subspace $\left\{\psi \in H_{\rho}^{2} ; L \psi=0\right\}$. It follows that the map $\mathcal{L}: H_{\rho}^{2} \rightarrow L_{\rho}^{2}$ defined by

$$
\mathcal{L} u:=\Pi u+L u
$$

is a one to one and onto map. We define $\mathcal{N}: H_{\rho}^{2} \rightarrow L_{\rho}^{2}$ by

$$
\mathcal{N}(u):=\Pi u+\mathcal{M}(u) .
$$

Then, $\mathcal{N}$ is analytic with $d \mathcal{N}(0)=\mathcal{L}$. Therefore, we obtain from the same argumentation as in [Si, Section 2] that there are constants $\theta \in(0,1 / 2)$ and $\sigma \in \mathbf{R}^{+}$such that if $u \in H_{\rho}^{2}$ with $|u|_{\rho} \leq \sigma$ then

$$
|\mathcal{M}(u)|_{2} \geq|\mathcal{E}(u)-\mathcal{E}(0)|^{1-\theta} \text {. }
$$

Let $|w(s)|_{\rho}<\sigma$ for $s \in\left[s_{1}, s_{2}\right]$. Then, by (3.11) and (3.17),

$$
\begin{aligned}
\frac{d}{d s}\{\mathcal{E}(w(s))-\mathcal{E}(0)\}^{\theta} & =\theta\{\mathcal{E}(w(s))-\mathcal{E}(0)\}^{\theta-1} \cdot\left(-\left|w_{s}\right|_{2}^{2}\right) \\
& =-\theta\{\mathcal{E}(w(s))-\mathcal{E}(0)\}^{\theta-1} \cdot\left|w_{s}\right|_{2}|\mathcal{M}(w(s))|_{2} \\
& \leq-\theta\left|w_{s}\right|_{2} \quad \text { for } \quad s \in\left[s_{1}, s_{2}\right]
\end{aligned}
$$


It follows that

$$
\left|w\left(s_{2}\right)-w\left(s_{1}\right)\right|_{2} \leq \theta^{-1} \int_{s_{1}}^{s_{2}}\left|w_{s}(s)\right|_{2} d s \leq \theta^{-1}\left\{\mathcal{E}\left(w\left(s_{1}\right)\right)-\mathcal{E}(0)\right\}^{\theta}
$$

Since $\mathcal{M}(0)=0$, we can verify that there exist constants $C_{j} \in \mathbf{R}^{+}$ $(1 \leq j \leq 3)$ such that for $s, \tau>0$

$$
\begin{gathered}
|w(s+\tau)|_{2} \leq \exp \left(C_{1} \tau\right)|w(s)|_{2}, \\
|w(s+\tau)|_{\rho} \leq C_{2}\left(1+\frac{1}{\tau}\right) \exp \left(C_{3} \tau\right)|w(s)|_{2} .
\end{gathered}
$$

By (3.19), (3.20), (3.21) and the assumption: $0 \in \omega(w(s))$, we obtain that $w(s) \rightarrow 0$ in $L_{\rho}^{2}$ (and also in $H_{\rho}^{2}$ ) as $s \rightarrow \infty$. Hence, $\omega(v(s))=\{\varphi\}$.

\section{ACKNOWLEDGMENTS}

The first version of this article was written while I was staying at Laboratoire d'Analyse Numérique, L'université de Paris-Sud. I would like to express my sincere gratitude to all members at the Laboratoire for their warm hospitality. I am very grateful to Professor Hiroki Tanabe for his constant encouragement, to Professor Takashi Suzuki for stimulating discussion, to Professors Yoshio Yamada, Ryuji Kajikiya and Thierry Cazenave for information on literature and to the referee for constructive comments. Remarks in Section 3 are due to the referee's suggestion.

\section{REFERENCES}

[CL] T. Cazenave and P. L. Lions, Solutions globales d'équations de la chaleur semi linéaires, Comm. Partial Differ. Equations, Vol. 9, 1984, pp. 955-978.

[D] E. DiBENEDETTO, Continuity of weak solutions to a general porous medium equation, Indiana Univ. Math. J., Vol. 32, 1983, pp. 83-118.

[EZ] EscoBEDo and ZuAZUA, Lare time behavior for convection - diffusion equations in $\mathbf{R}^{\mathrm{N}}$, J. Func. Anal., Vol. 100, 1991, pp. 119-161.

[F] H. Fusita, On the blowing up of solutions of the Cauchy problem for $u_{t}=\Delta u+u^{1+a}$, J. Fac. Sci. Univ. Tokyo Sect. 1, Vol. 13, 1966, pp. 109-124.

[G] Y. GigA, A bound for global solutions of semilinear heat equations, Comm. Math. Phys., Vol. 103, 1986, pp. 415-421.

[GS] B. GIDAS and J. SPRUCK, A priori bounds for positive solutions of nonlinear elliptic equations, Comm. P. D. E., Vol. 6, 1981, pp. 883-901.

[HW] A. HARAUX and F. B. WeISSLER, Non-uniqueness for a semilinear initial value problem, Indiana Univ. Math. J., Vol. 31, 1982, pp. 167-189. 
[Kavi] O. KAVIAN, Remarks on the large time behavior of a nonlinear diffusion equation, Annal. Institut Henri Poincaré - Analyse non linéaire, Vol. 4, 1987, pp. 423-452.

[Kawa] T. KaWANAGO, Existence and behavior of solutions for $u_{t}=\Delta\left(u^{m}\right)+u^{l}$, Preprint.

[Le] H. A. LEVINE, The role of critical exponents in blowup theorems, SIAM Review, Vol. 32, 1990, pp. 262-288.

[Li] P. L. LIons, Asymptotic behavior of some nonlinear heat equations, Physica 5D, 1982, pp. 293-306.

[LN] T. Y. LeE and W. M. NI, Global existence, large time behavior and life span of solutions of semilinear parabolic Cauchy problem, Trans. Amer. Math. Soc., Vol. 333, 1992, pp. 365-371.

[NST] W. M. NI, P. E. SACKS and J. TAVATZIS, On the asymptotic behavior of solutions of certain quasilinear equation of parabolic type, J. Differ. Equations, Vol. 54, 1984, pp. $97-120$.

[S] P. E. SACKS, Continuity of solutions of a singular parabolic equation, Nonlinear Anal., Vol. 7, 1983, pp. 387-404.

[Si] L. SimON, Asymptotics for a class of non-linear evolution equations, with applications to geometric problem, Annals Math., Vol. 118, 1983, pp. 525-571.

[W] X. WANG, On the Cauchy problem for reaction - diffusion equations, Trans. Amer. Math. Soc., Vol. 337, 1993, pp. 549-590.

(Manuscript received March 18, 1994; revised November 24, 1994.) 